\title{
JouFLU: upgrades to the Fiber Linked Unit for Optical Recombination (FLUOR) interferometric beam combiner.
}

\author{
N. J. Scott ${ }^{a}$, E. Lhomé ${ }^{c}$, T. A. ten Brummelaar ${ }^{a}$, V. Coudé du Foresto ${ }^{c d}$, R. Millan-Gabet ${ }^{b}$, J. \\ Sturmann $^{a}$, and L. Sturmann ${ }^{a}$ \\ ${ }^{a}$ Georgia State University/The CHARA Array, Mount Wilson Observatory, Mount Wilson, \\ CA 91023, USA \\ ${ }^{b}$ California Institute of Technology, NASA Exoplanet Science Institute, Pasadena, CA 91125, \\ USA \\ ${ }^{c}$ LESIA - CNRS, Observatoire de Paris, 92192 Meudon Cedex, France \\ ${ }^{d}$ Visiting scientist, Center for Space and Habitability, Bern University, Switzerland
}

\begin{abstract}
The Fiber Linked Unit for Optical Recombination (FLUOR) is a precision interferometric beam combiner operating at the CHARA Array on Mt. Wilson, CA. It has recently been upgraded as part of a mission known as "Jouvence of FLUOR" or JouFLU. As part of this program JouFLU has new mechanic stages and optical payloads, new alignment systems, and new command/control software. Furthermore, new capabilities have been implemented such as a Fourier Transform Spectrograph (FTS) mode and spectral dispersion mode. These upgrades provide new capabilities to JouFLU as well as improving statistical precision and increasing observing efficiency. With these new systems, measurements of interferometric visibility to the level of $0.1 \%$ precision are expected on targets as faint as 6th magnitude in the $\mathrm{K}$ band. Here we detail the upgrades of JouFLU and report on its current status.
\end{abstract}

Keywords: Instrumentation: interferometers - techniques: high angular resolution - techniques: interferometric

\section{INTRODUCTION}

Georgia State University's Center for High Angular Resolution Astronomy currently operates a long baseline optical interferometer known as the CHARA Array at Mt. Wilson Observatory in California. ${ }^{1}$ The array is made up of six one-meter telescopes in a Y-configuration (see Figure 1). These telescopes provide baselines from $34 \mathrm{~m}$ to $331 \mathrm{~m}$ for beam combiners that operate in both the optical and infrared wavebands. The CHARA Array has a maximum resolving power of 200 micro-arcseconds in the visible wave band. The light from each telescope is conveyed in vacuum to a central Beam Combining Facility. In this $100 \mathrm{~m}$ long building the individual beams pass through a complex system of motorized carts and optics to ensure that each beam travels the same distance in order to keep zero Optical Path Difference (OPD) throughout the night. Once the path lengths are equalized the beams may be passed to any of the six beam combiners in the Beam Combining Lab that perform measurements of fringe visibility, phase, or spectrum.

One of the first beam combiners in operation at CHARA was FLUOR, near-infrared interferometric beam combiner built by the Laboratoire d'Etudes Spatiales et d'Instumentation en Astrophysique (LESIA) of the Observatoire de Paris. Beams from any two CHARA telescopes can be directed to FLUOR which gives it access to 15 separate baselines. Originally setup on Kitt Peak, Arizona in 1992, FLUOR moved to the Infrared and Optical Telescope Array (IOTA) on Mt. Hopkins in 1995. In 2002 FLUOR moved to the CHARA Array.

In recent years it became clear that several improvements could increase the efficiency, improve the throughput, and better integrate FLUOR with the CHARA Array. The Jouvence of FLUOR (JouFLU), loosely translated as 'rejuvenation' of FLUOR, project has replaced much of the optical bench setup of FLUOR to provide

Further author information: (Send correspondence to N. J. Scott) E-mail: nic@chara-array.org, Telephone: 1 (626) 796-3730

Optical and Infrared Interferometry IV, edited by Jayadev K. Rajagopal, Michelle J. Creech-Eakman,

Fabien Malbet, Proc. of SPIE Vol. 9146, 91461A • C) 2014 SPIE • CCC code: 0277-786X/14/\$18

doi: 10.1117/12.2057129 Proc. of SPIE Vol. 9146

Proc. of SPIE Vol. $914691461 \mathrm{~A}-1$ 


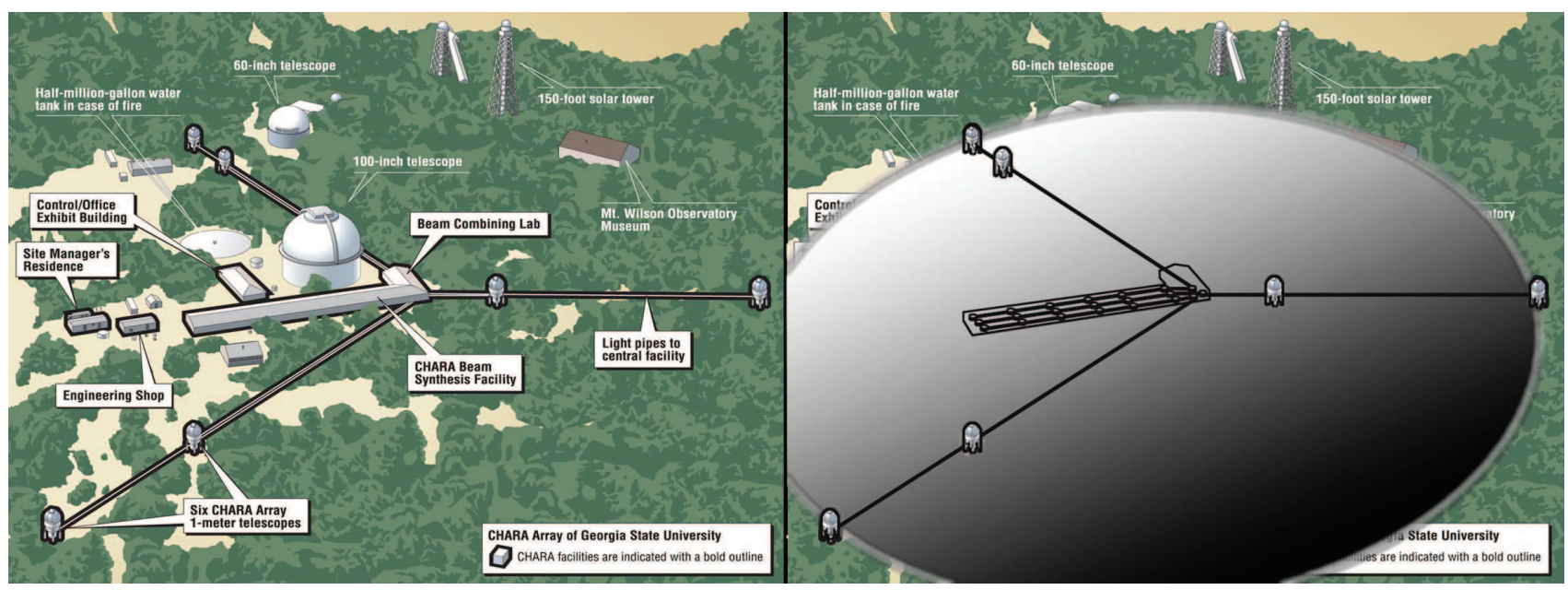

Figure 1. Layout of the CHARA Array's six telescopes, light pipes, and Beam Combining Lab within the context of the other facilities on Mt. Wilson (left). The illustration on the right shows the size of a mirror of equivalent resolving power to the CHARA Array. Also visible within the outline of the Beam Combining Lab are the "Pipes of Pan" (PoPs). Mirrors inserted at these points allow for various fixed intervals of large delay.

greater opto-mechanical stability. ${ }^{2}$ This includes new motorized mounts for the mirrors that feed light into the optical fibers, new high precision motorized stages which control the optical path length to generate fringes (OPD SCAN and OPD STAT), an infrared pupil plane camera system, a visible light alignment camera, and improvements to the science camera (NICMOS). Figure 2 shows the extent of the changes to the optical bench setup. Alignments that were previously done in the lab by hand and with an alignment telescope before the night's observations began are now automated. In addition to the hardware upgrades, a concurrent replacement of the control software has produced an entirely new software system compliant with the CHARA operating environment, enabling JouFLU to be maintained at the leading edge of improvements to other combiners at the array. As another combiner receives relevant new software features or tools, JouFLU may now benefit from them too with a minimum of effort. From these software changes FLUOR now has remote operation capability, potential for greater science data throughput, and higher statistical precision.
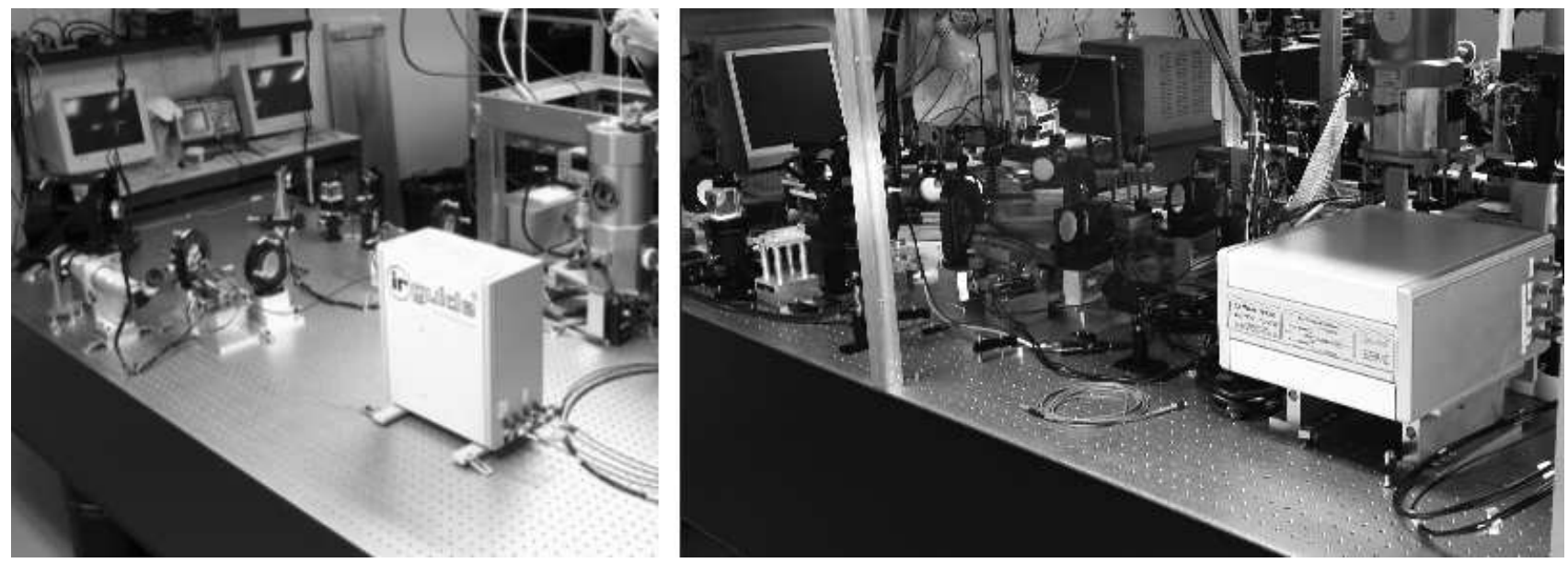

Figure 2. FLUOR shortly after its move to the CHARA Array left. JouFLU in its current condition in the CHARA lab right. 


\section{THE INSTRUMENT}

FLUOR is a two-way infrared interferometric beam combiner operating in the $\mathrm{K}^{\prime}$ band $(\lambda=2.20 \mu \mathrm{m}, \Delta \lambda=$ $0.40 \mathrm{FWHM})$. To produce visibility measurements with a very high precision $\left(\mathrm{V}^{2} \approx 1 \%\right)$ FLUOR utilizes the spatial filtering properties of optical fibers. ${ }^{3}$ Such precision allows FLUOR to make measurements of scientifically interesting features such as stellar radii accurate to an error level of one percent or less. The instrument is highly suited to the studies of debris disks ${ }^{4}$ and exozodical dust, ${ }^{5}$ young star circumstellar disks, ${ }^{6}$ Cepheid variable stars and Baade-Wesselink distance measures,${ }^{7}$ binary star studies, ${ }^{8}$ astereoseismology,${ }^{9}$ and high dynamic range sources ${ }^{10}$ requiring observations at dynamic ranges of $10^{2}$ to $10^{6}$.

\subsection{MOTION CONTROL AND THE OPD STAGES}

In the original FLUOR setup one of the two beams first encountered a mirror mounted on a piezo stack dither stage. This has been replaced on the JouFLU optical bench. Now each of the two beams first encounter an OPD stage which has a payload consisting of a set of two mirrors at $45^{\circ}$ to the beam path (see Figure 3). JouFLU interference fringes are produced by temporal OPD modulation of one of these stages (OPD SCAN: XMS50 stage). The reliable and accurate motion of this stage is critical to the performance of JouFLU. In addition to modulation of fringes and adjusting the OPD, several stages were required for the JouFLU improvements. The addition of a Newport XPS Series Universal High-Performance Motion Controller/Driver has enabled the use of multiple mechanized stages to offer various configurations of the optical bench. We have connected 7 Newport stages to the XPS controller. There is a stage for moving the FTS beamsplitter into position (FTS: UTS100PP), two OPD stages (the previously mentioned OPD SCAN: XMS50 and OPD STAT: UTS100PP), two alignment stages (ALIU A and ALIU B: $2 \times$ UTS150PP), a stage to adjust the focus for the H-band pupil camera (ALIU L2: MFA-PPD), and the OUTPUT optics stage (OUT: M-URM100PP). The two Optical Path Difference (OPD) stages perform different functions: OPD SCAN is a dynamic scanning stage which modulates the OPD and generates fringes within MONA while OPD STAT is an adjustable static stage to correct residual OPD. These stages carry an identical payload consisting of a pair of mirrors.

The scanning stage meets rigid requirements for linear velocity stability over its full range of travel. This stage was tested for stability using laser metrology prior to installation and achieves $<1 \%$ error in its velocity at $100 \mu \mathrm{m} / \mathrm{s}$ (see Figure 4). In addition, further custom tuning was performed by a Newport technician. The stage is powered by a linear DC motor and has $50 \mathrm{~mm}$ of travel. The greater range of travel for this new stage greatly surpasses the $200 \mu \mathrm{m}$ of the FLUOR piezoelectric dither mirror. The increased range is necessary for the use of FTS mode. During normal observation mode and collecting fringes at $100 \mathrm{~Hz}$ the scanning stage travels at $105 \mu \mathrm{m} / \mathrm{s}$ (half the optical path velocity due to double pass) over a range of $150 \mu \mathrm{m}$. For FTS mode the stage travel range must be 10 times this. The exact velocity of the OPD SCAN stage is determined by the NICMOS camera readout frequency. Fringes are temporally modulated and scanned at a rate of 5 samples per fringe (2.5 times Nyquist). So for $100 \mathrm{~Hz}$ fringes NICMOS reads out at $500 \mathrm{~Hz}(2 \mathrm{~ms})$. The XPS Motion Controller has been programmed to send a signal to the JouFLU control computer to report when the OPD SCAN stage is moving at a constant velocity. The JouFLU computer then only requests data from NICMOS when this is true. This ensures that the fringes are only recorded under the constant velocity situation and not when the stage is accelerating. Alternatively, it is also possible to collect data without the signal from XPS by using software timing delays based off measurements of the camera readout rate.

While the motion of the OPD SCAN stage is the most critical moving part on the JouFLU bench, several other stages are involved in the operation of JouFLU. While OPD STAT does not have the strict velocity requirements of OPD SCAN, it does need a longer range of travel. This stage corrects for the offset created by introduction of FTS beam splitter. Approximately 4 centimeters of path difference are introduced in FTS mode due to the separation of the two beam paths. The OUTPUT stage of JouFLU is a motorized rotating circular platform. It has three configurations: an open position for normal observations, a red LED for retro-injecting light for alignment procedures, and a ZnSe biprism to produce spectrally dispersed fringes. The last important set of stages is the pair of ALIgnment Units (ALIU), these will be discussed in section 2.5.

The integrated interface and ethernet control of the XPS provides the ability to correct alignments or reconfigure the instrument while on sky without going into the lab and opens the possibility for completely remote

Proc. of SPIE Vol. $914691461 \mathrm{~A}-3$ 


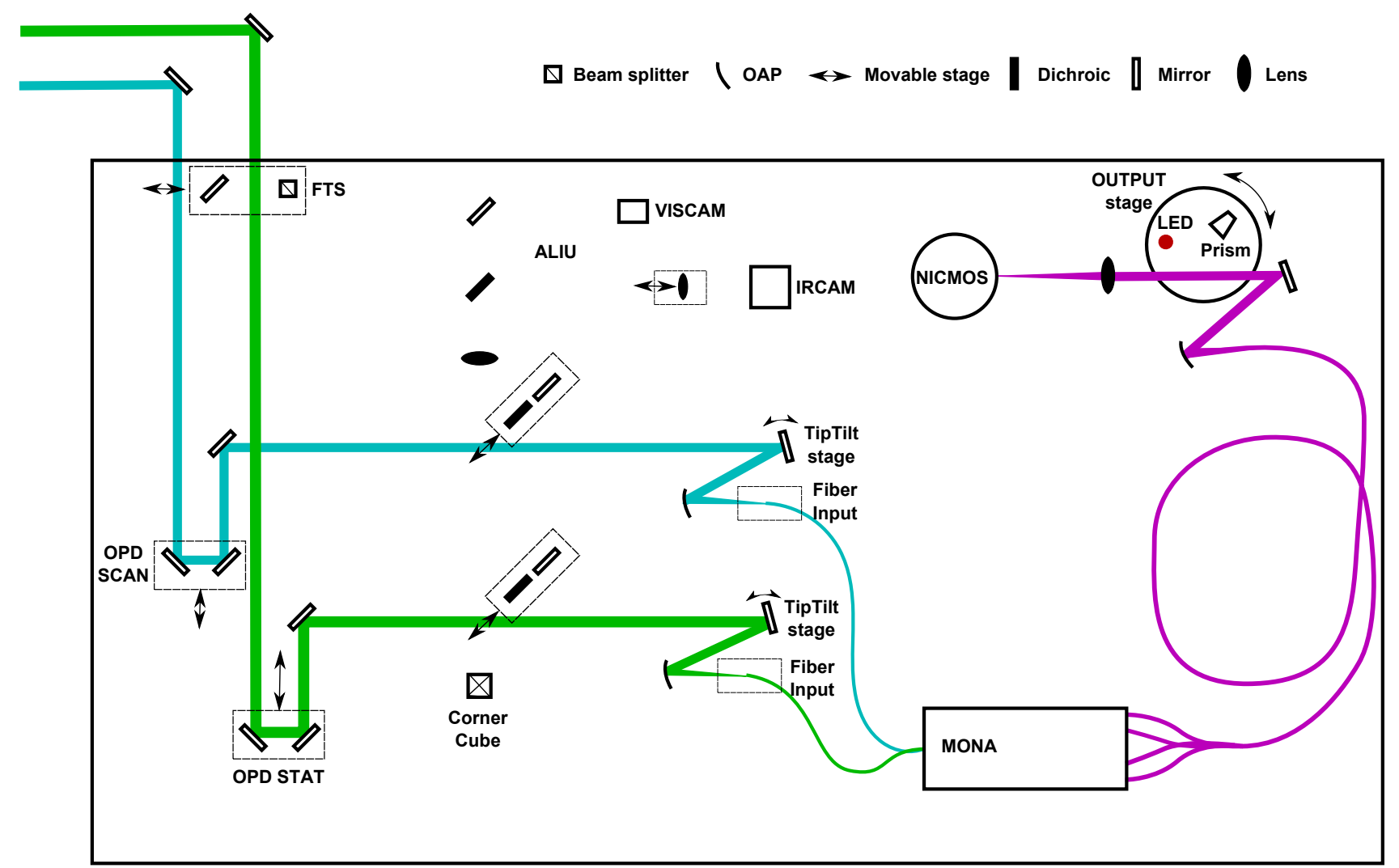

Figure 3. JouFLU in the configuration used when taking observations. The alignment stages are out of the path of the beam and the OUTPUT stage is moved to the open position. Also shown for completeness are the optics of the alignment portion of the bench, see Figure 9 for their use. The Fourier Transform Spectrograph beamsplitter is removed from the beam path.

operations from any of the CHARA Remote Observing Control Rooms located in Atlanta, Meudon, Nice, Sydney, Ann Arbor, and Bonn.

\subsection{FIBER INJECTION}

After passing the OPD stages each beam needs to be injected into the fibers. The fiber injection system of FLUOR has been greatly improved by the replacement of the stepper motors controlling the tip/tilt of the fold mirror by Zaber model T-MM stages. The previous FLUOR stepper motors were not precise enough to produce repeatable alignment for fiber injection. The minimum step size of these stages is 310 milliarcseconds or $1 / 20$ of the $6.5 \mu \mathrm{m}$ core diameter of the fibers. In practice a step size of $\approx 3-6 \mu \mathrm{m}$ is used to improve repeatability of raster scans. This is a large improvement over the replaced stepper motors which had a step size roughly equal to the fiber core diameter with poor repeatability. The higher precision injection of light allows for accurate raster scanning to maximize the amount of light that reaches MONA. The Zaber tip/tilt stages are also much faster than the previous stepper motors which allows for more rapid and larger raster scans. This improvement in precision shifts the major limiting factor for efficiency of light injection from the quality of the raster alignment to the atmospheric seeing conditions. In practice, a $r_{0}$ value of $5 \mathrm{~cm}$ or greater results in the majority of light to be incident on the fiber core. Figure 5 shows the results and analysis of a typical raster scan.

These Zabers direct the light onto a two inch diameter gold coated f/1.3 $30^{\circ}$ Off-Axis Parabola (OAP) for each beam. The OAPs focus the light onto fiber injection stages capable of XYZ translation. The optical fibers are terminated in high precision E-2000 fiber connectors which allow the fibers to be unplugged and replugged without loss of alignment. 


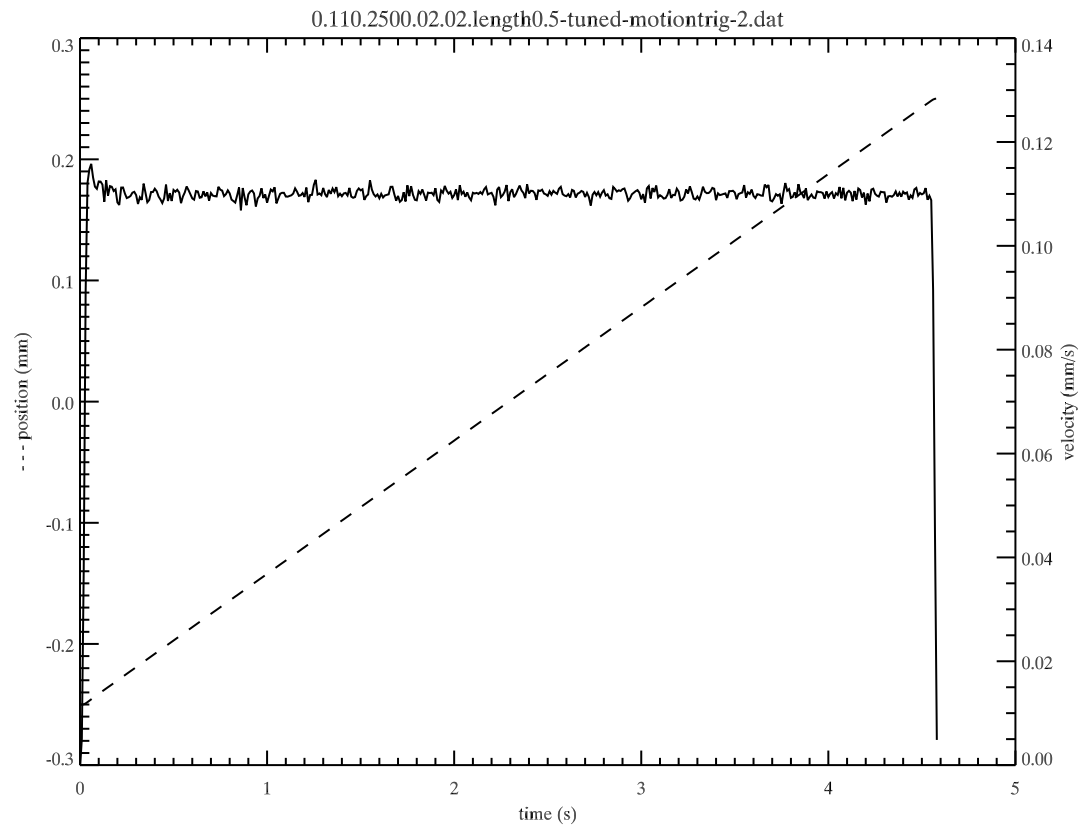

Figure 4. A $110 \mu \mathrm{m} / \mathrm{s}$ velocity scan of the OPD SCAN stage for a length of $5 \mathrm{~mm}$ gives a rms error of $1.3 \%$ over its full range. However most of this error is at the start and end of motion. To minimize this effect the camera data is taken after the stage motion has stabilized. This results in an rms error $<1 \%$.

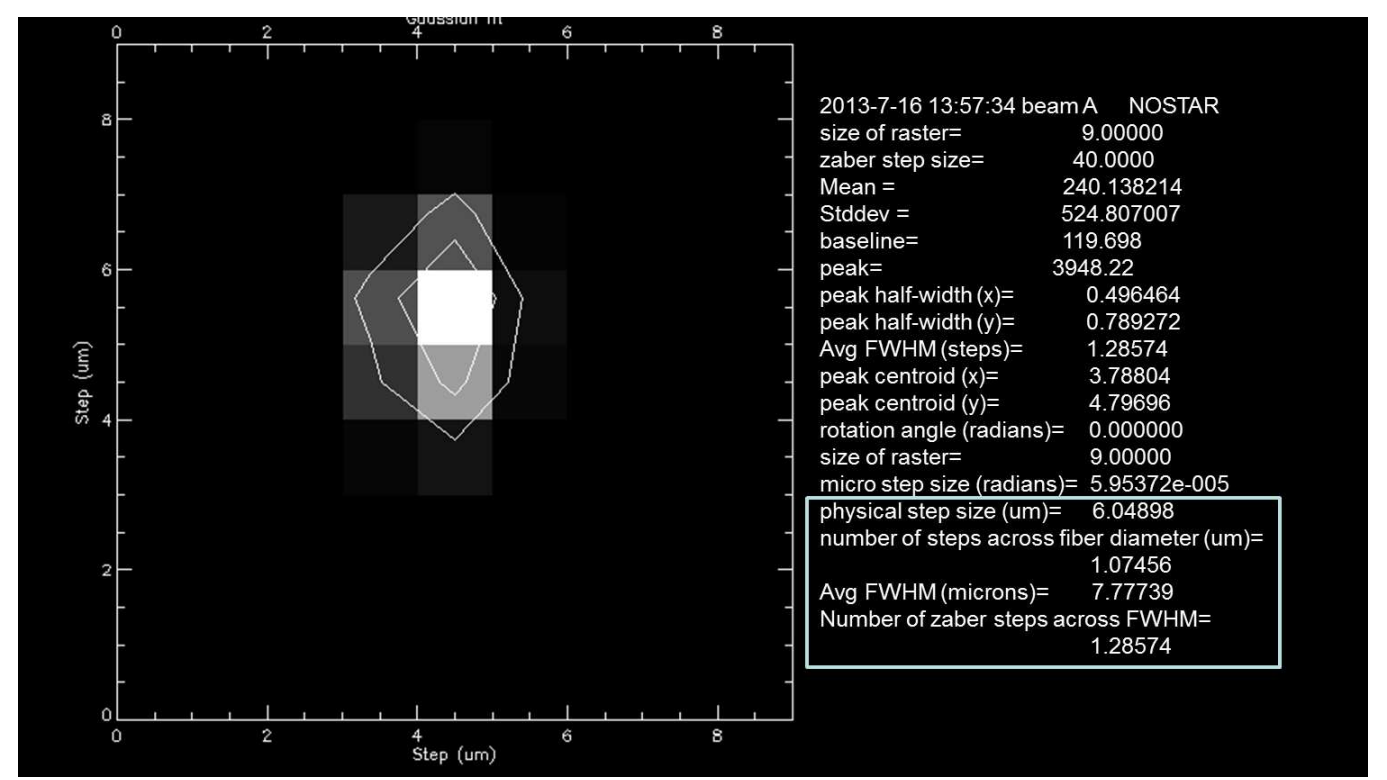

Figure 5. A typical raster performed with the Zaber stepper motors to inject light onto the Off-Axis Parabolas (OAPs) and into the $6.5 \mu \mathrm{m}$ fibers. For most circumstances a step size of $1 / 2$ or equal to the fiber core diameter is used. The contour lines overplot a Gaussian fit to the recorded raster scan, the details of the fit and its relation to the fiber core are given on the right. 


\subsection{BEAM COMBINATION}

In FLUOR, beam combination occurs within optical fiber couplers. The fiber couplers are located inside a closed system called "MONA" built by Le Verre Fluoré. Two injection stages (Fiber input in Figure 3) feed light into two Y-fiber couplers which in turn feed one of their outputs into a X-fiber coupler (See Figure 6). Interferometric combination occurs in the X-fiber coupler. MONA outputs two photometric channels (PA and PB) from the Y-fiber couplers and two interferometric signal channels (S1 and S2) from the X-coupler. All four fibers join into a single fiber bundle in a $125 \mu \mathrm{m}$ square pattern. This bundle connects to another fiber translation stage and OAP. After the OAP the light enters an objective which images the fiber bundle onto the NICMOS science camera which is read out as four regions of interest. Typically this is four single pixels, one for each fiber in the bundle. The difference is taken between the two interferometric pixels to increase the Signal-to-Noise Ratio (SNR) while the photometric channels are recorded simultaneously for data calibration during the reduction stage. ${ }^{3}$

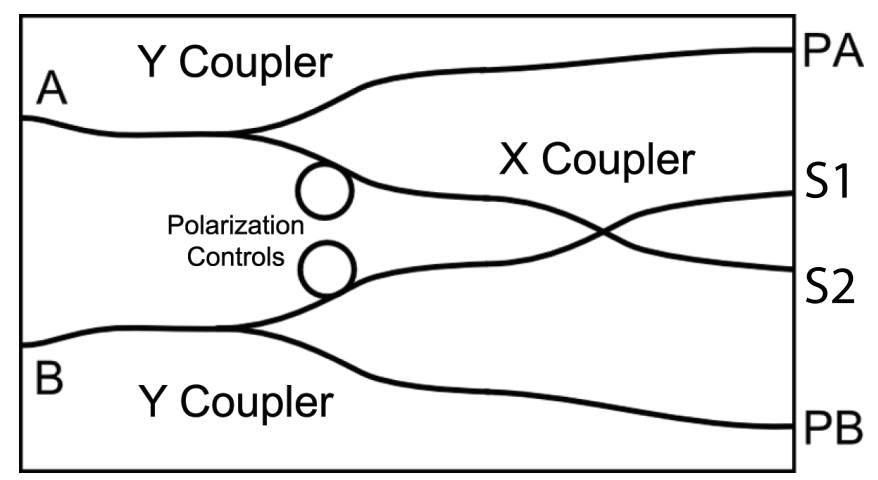

Figure 6. The MONA fiber beam combiner consists of two Y-fiber couplers and one X-fiber coupler outputting one photometric channel for each beam and two interferometric channels total. Fiber polarization can be adjusted by changing the amount of bend to the fiber in the two loops.

As part of the JouFLU project the fiber combiner box, MONA, was sent to Le Verre Fluoré for re-calibration and adjustment. The fiber heads were cleaned and two knobs with numbered scales were added to provide adjustment of the polarization of the fibers. Once installed back at the CHARA Array, interferometric signal throughput was maximized. This was achieved by scanning through the range of the polarization adjustment for each beam while measuring the visibility of lab fringes generated by a controlled white light source (see Figure 7).

\subsection{THE CAMERA, NICMOS}

The four outputs (two interferometric, two photometric) of the MONA combiner are imaged onto four pixels of a NICMOS3 array, housed in a camera originally developed for the IOTA interferometer. ${ }^{11}$ We use the same dewar, readout electronics and control software approach as in the original implementation. In 2007 however, the original NICMOS3 array failed, and was replaced by another engineering grade NICMOS3 array, kindly loaned by NOAO. Although the replacement array has a larger number of bad pixels, which can easily be avoided, the noise characteristics remain similar. Camera control has been integrated into the CHARA environment as described in Section 2.8. The main JouFLU CPU coordinates the Newport XPS and the MS-DOS machine that communicates with NICMOS. The Newport XPS directly triggers reading of the camera so that data is only collected when the fringe scanning stage (OPD SCAN) is moving with constant velocity. These data are then sent to the JouFLU computer for real time display and recording.

In Figure 8 we show the results of updated measurements of the camera gain and readout noise, performed using the standard method of measuring the flux-variance curve, and using the same readout mode as is used for on-sky observations of the four target pixels. As in previous implementations, we use Fowler sampling for noise reduction: the array is reset for each sample, after which the pixels of interest are sampled continuously as they discharge. ${ }^{12}$ The integration time for each recorded data point is set by the time needed to sample each target pixel, possibly multiple times. For a given camera read frequency an optimum number of reads and loops for 


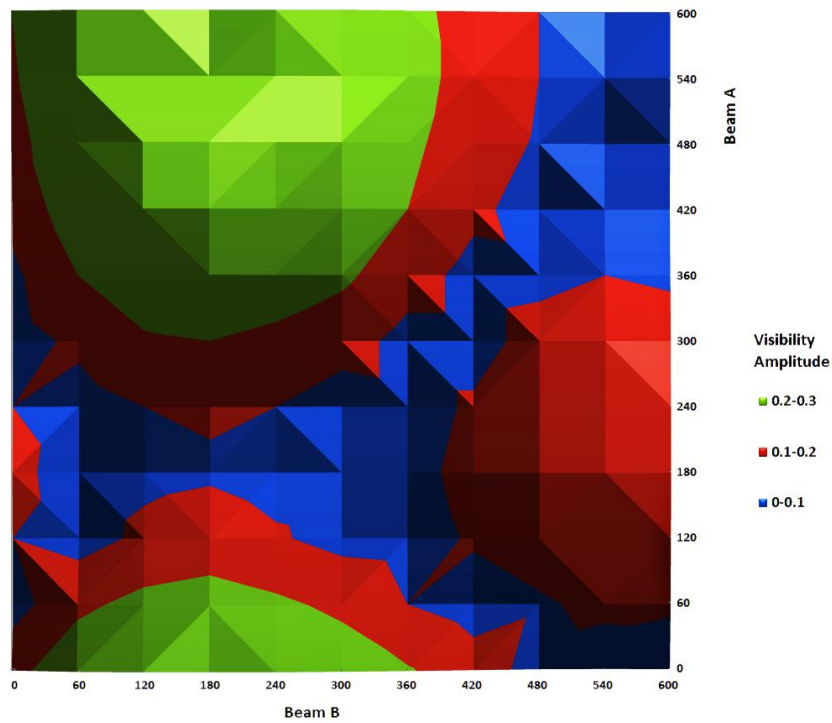

Figure 7. The effects of adjustment of the fiber polarization for each of the two beams on white light fringe visibility. Each fiber has a knob which affects the bend of the fiber. Each knob was incrementally adjusted in steps of 60 arbitrary units to give 100 data points. Based on these results MONA was set to 480 and 180 for the top and bottom knobs, respectively.

the pixels is determined to give the correct sample time and minimize read noise. For example, to get a $500 \mathrm{~Hz}$ camera readout, the four pixels are read 11 times in one loop. This combination was found to give a 2 ms readout with minimal readout noise. The timing of pixel readout for various modes was checked by oscilloscope.

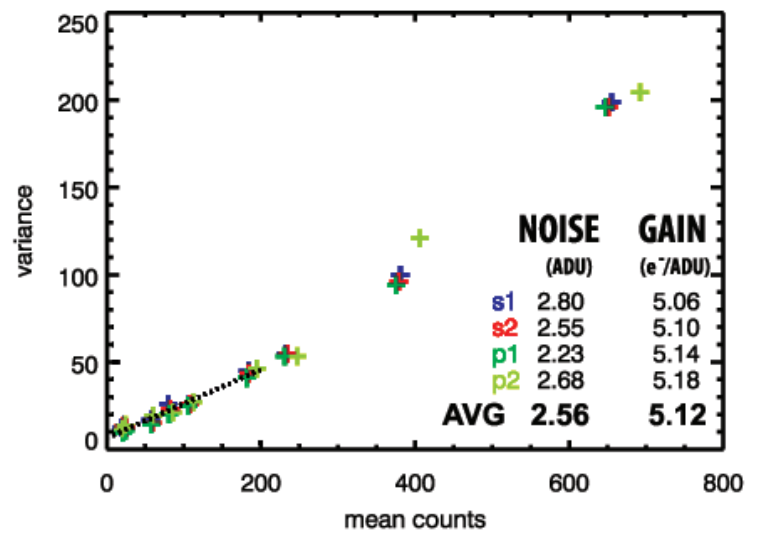

Figure 8. The results of gain and readout noise tests of the NICMOS camera for each of the four read pixels. The camera was operated in destructive mode at $500 \mathrm{~Hz}$ with 2 loops and 3 reads of each pixel. Mean counts and variance were recorded as light levels were incrementally increased. The first 200 counts were treated as linear and a regression was performed to determine the gain. Mean readout noise for the four pixels read is 2.56 ADU with a mean gain of 5.12 $e^{-} / \mathrm{ADU}$.

\subsection{ALIGNMENT SYSTEMS}

A major focus of JouFLU is to improve the quality and ease of optical alignment procedures to increase the observing efficiency of FLUOR. Previously with FLUOR, optical alignments had to be performed by personnel inside the lab prior to an observing night. The addition of ALIU improves the accuracy and repeatability of alignment for the beams. These stages also add the option to view either the image or pupil planes. ALIU provides a method for reliable alignment adjustments to be performed during the night with little interruption 
of data collection, or to be performed remotely. ALIU consists of two long travel Newport stages, one for each beam, which carry a payload of a mirror and a dichroic. These stages are placed at $45^{\circ}$ to the beam path and have three set positions: Open, Dichroic, and Mirror. During science observing ALIU is clear of the beam path. When an alignment needs to be performed, one of the stages can position the dichroic into the path of the beam that is to be aligned. The OUTPUT stage can retro-inject light from a red LED thought the beam combiner, MONA. After leaving the fibers the light from the LED hits the ALIU dichroic and is reflected to a corner cube. The corner cube directs the light to pass through the dichroic and to another system consisting of a mirror and focusing lenses (see Figure 9). The LED spot is imaged by a Prosilica visible light camera. The position of this spot is compared with that of a green laser spot produced by CHARA. The motorized tip/tilt stages can then overlay the two spots to conjugate the system.

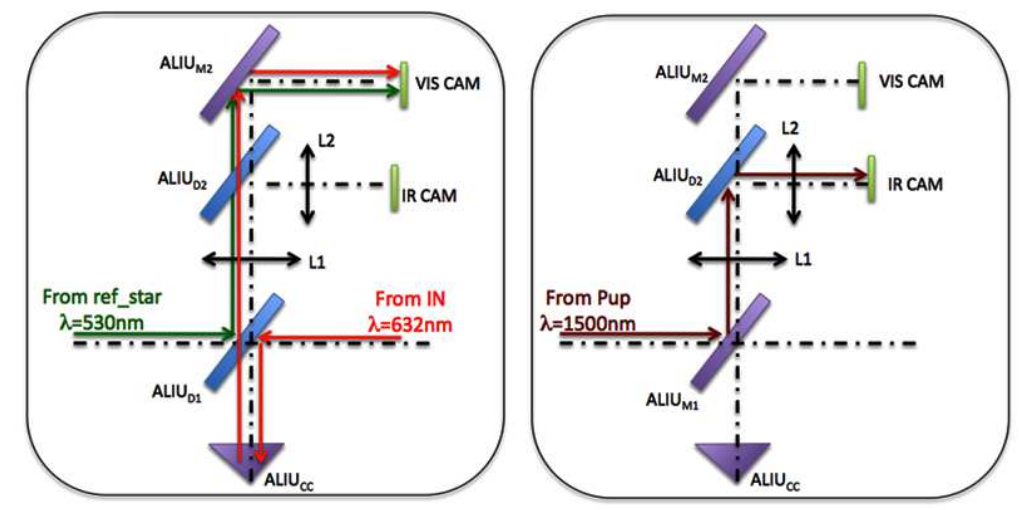

Figure 9. FLUOR in the configuration used when aligning with the ALIU system and the visible camera (VISCAM). The ALIU stage for the desired beam is moved to the dichroic position and the light from the external source or star passes through a focusing lens (L1) and another dichroic (D2) to reach a fold mirror (M2) which directs it to the alignment camera (left). To check the CHARA pupil for vignetting or other possible loss of flux an ALIU stage is moved to the mirror position and external source light is passed through the same lens used by the VISCAM, reflects off of dichroic D2, and reaches a focusing lens mounted on a stage (L2). The CHARA pupil is then recorded with the infrared camera (right). (image credit: LESIA - CNRS/Observatoire de Paris)

Another available option is the pupil viewing mode. To utilize the pupil camera either of the ALIU stages move to the mirror position. The beam passes through a lens and is then redirected at a right angle with a dichroic. It passes through the two notch filters and a focusing lens. The use of $1.319 \mu \mathrm{m}$ delay line metrology laser by the array necessitated the inclusion of two notch filters to prevent possible damage to the pupil plane camera. This pupil camera is an InGaAs detector operating in the $\mathrm{H}$ band $(0.9-1.7 \mu \mathrm{m})$ and is situated next to the visible alignment camera. It allows problems such as vignetting and other issues leading to a loss of flux to be diagnosed during on-sky operations. This camera is a commercially available electronically cooled $320 \times 256$ InGaAs detector. The global response function for this system is shown in Figure 10.

\subsection{SPECTRAL DISPERSION MODE}

The inclusion of a low dispersion $(\mathrm{R} \approx 70)$ biprism on the OUTPUT stage of JouFLU allows for the measurement of dispersed fringes across 5 channels. Figure 12 shows the camera display during spectrally dispersed mode. Each rectangular strip corresponds to a single spectrally dispersed output of MONA (clockwise from the top left: PA, S2, PB, and S1). Data from fringe scanning mode is shown in Figure 11. The number of channels is currently limited by the readout rate of the camera. Four regions of 5 pixels each is currently the maximum possible at $500 \mathrm{~Hz}$. The simultaneous measurement of fringes will increase the statistical accuracy of the resulting measurement for sources bright enough to not be dominated by detector noise. The spectral dispersion mode will be of particular use when the science star and the calibrator star are of different spectral types. The measurement of visibilities in multiple wavelengths will enable the removal of chromatic bias/bandwidth smearing. Figure 13 shows the wavelength of each channel. 


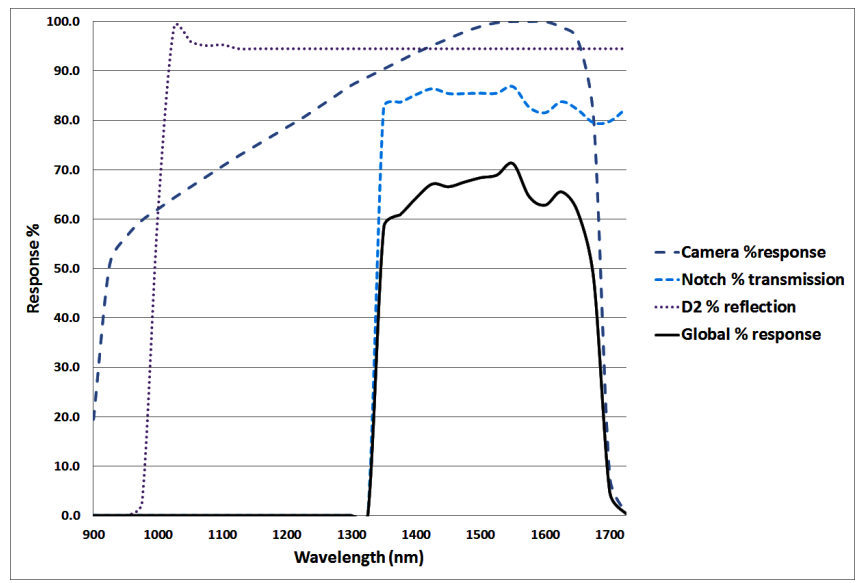

Figure 10. The global response function for the H-band pupil imaging camera. Also plotted are the response functions for a single notch filter, the camera response function, and the dichroic (D2) reflection function. The global function is the product of the camera's response, the two notch filters, and the D2 reflection.

Another use of spectral dispersion mode is for the recording of differential phase. Normally with two beam interferometry no information of the phase is available. However, by measuring across multiple wavelengths the relative phase difference between wavelengths can be obtained. As a feasibility study of this technique, spectrally dispersed fringes were recorded in 2004. Spectral dispersion mode was implemented in Fall 2013 with first spectrally dispersed fringes obtained on sky in early 2014.

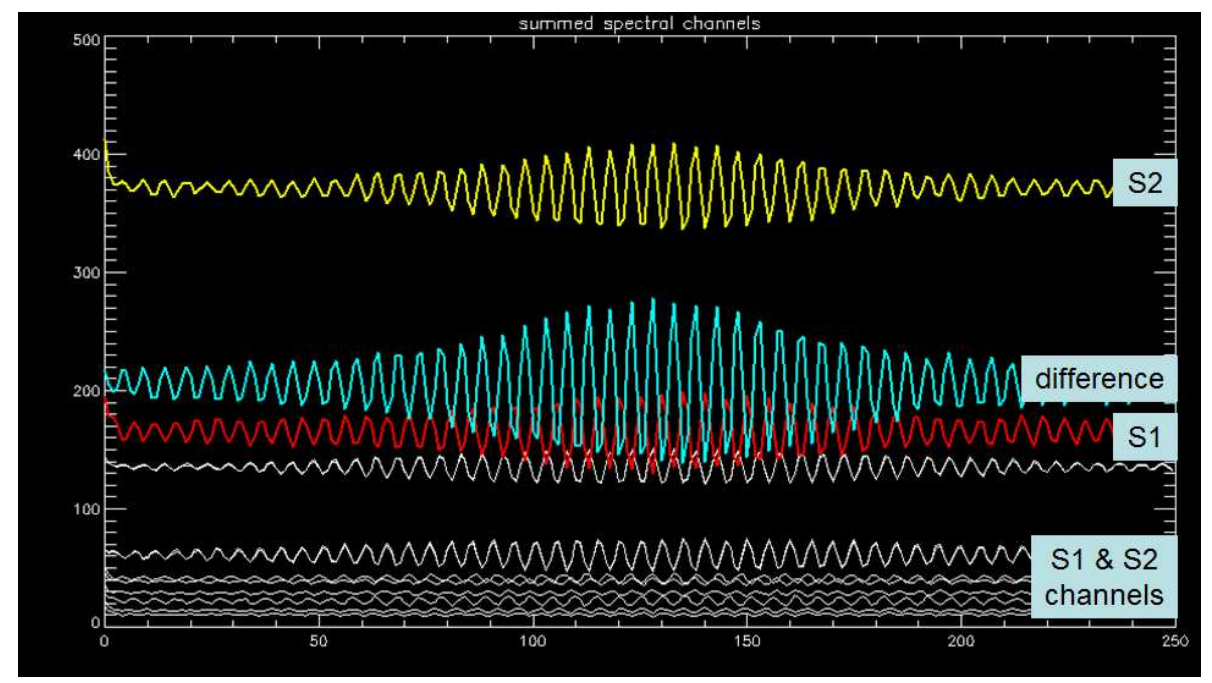

Figure 11. Fringes recorded from the lab white light source. The bottom 10 scans are from each pixel in the dispersed interferometric channels. The fringes labeled 'S1' and 'S2' are the sums of the 5 pixels in each channel. The large middle fringe is the difference between the two summed channels.

\subsection{FOURIER TRANSFORM SPECTROGRAPH}

The addition of a Fourier Transform Spectrograph (FTS) mode to JouFLU provides wavelength calibration of the science camera. In FTS mode a single beam is taken from CHARA. The single beam does not suffer from differential seeing and atmospheric piston. This allows longer scans at $100 \mathrm{~Hz}$. The single beam is passed through a beamsplitter and fold mirror mounted on a motorized stage. The beamsplitter generates the two beams necessary to feed into MONA for combination. As in other modes, the OPD SCAN stage modulates the Optical Path Difference (OPD). The resulting interferogram is affected only by the spectrum instead of spatial structure of the source. This spectrum can then be used to provide photometric and spectral calibrations of the 


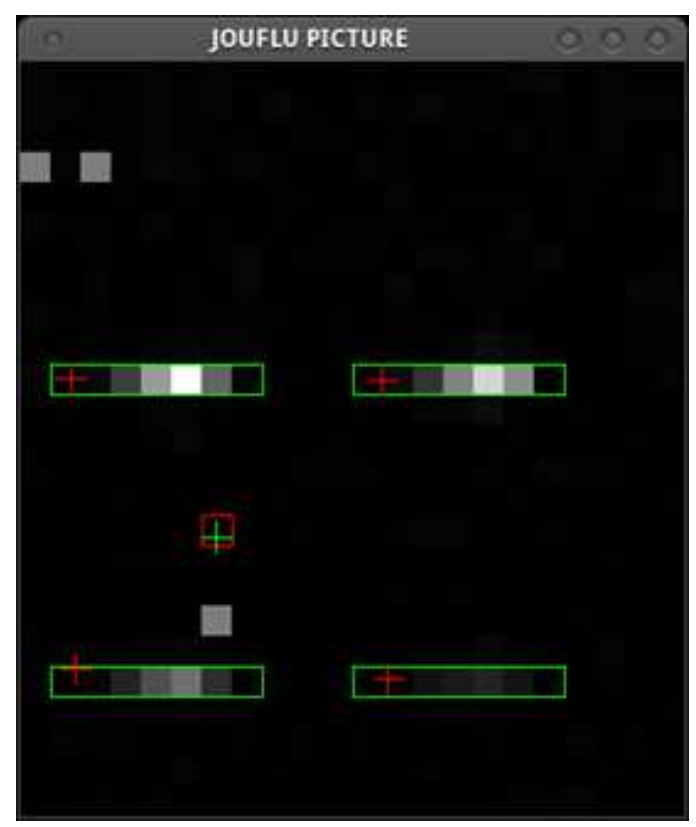

Figure 12. The camera display used during camera alignments and to check the amount of flux. Shown here during spectral dispersion mode the four MONA outputs are displayed in rectangular boxes (clockwise from the top left: PA, $\mathrm{S} 2, \mathrm{~PB}$, and $\mathrm{S} 1$ ). The cross-hairs function as aids during camera alignment.

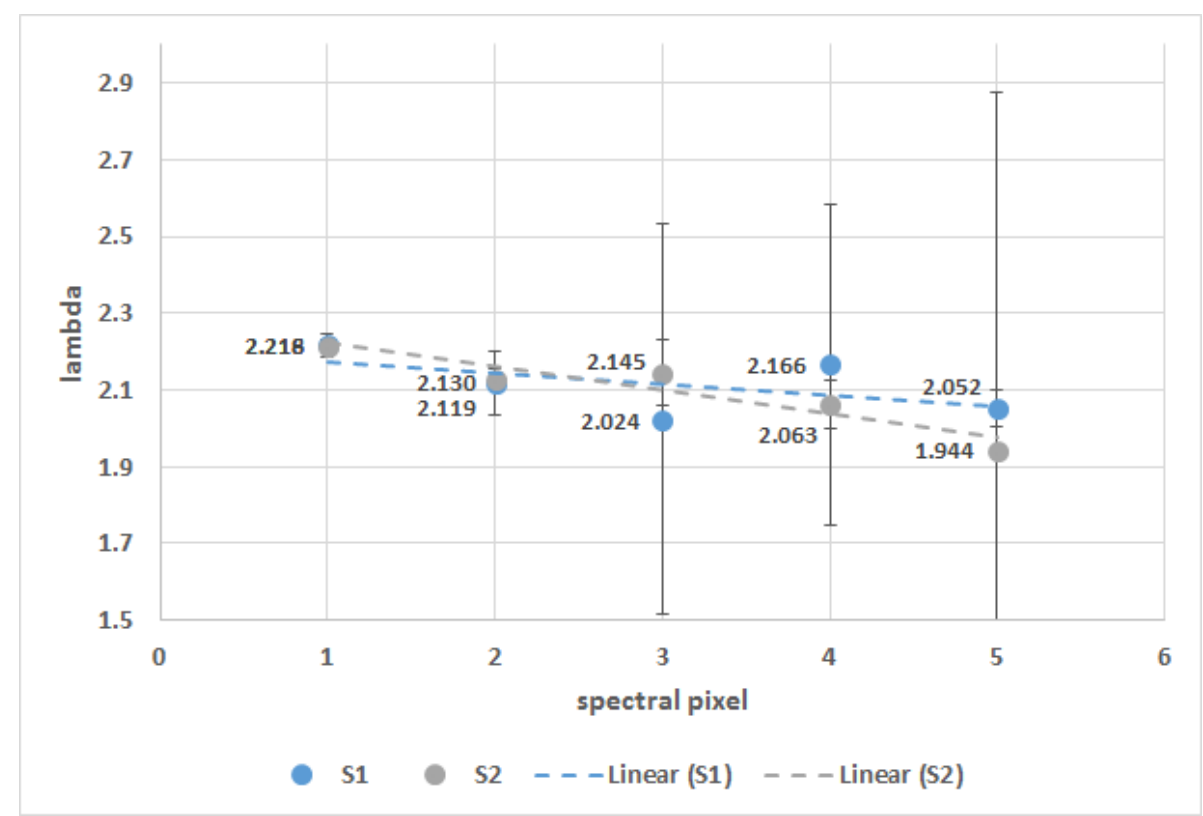

Figure 13. The wavelength for each pixel of the interferometric channels was calculated by fringe fitting lab fringe data. Mean $\mathrm{R}=9.8$, mean channel bandwidth $=0.22 \mu \mathrm{m}$. The large error bars in the higher numbered pixels is due to low flux from the lab source. 
instrument. This mode is currently undergoing tests in the lab and is expected to be tested on sky later this year.

\subsection{SOFTWARE}

The previous Command/Control system for FLUOR consisted of software originally written for use while FLUOR was at IOTA. The software was written in LabView and operated parallel to the normal functions of CHARA. As such, it was unable to take advantage of some of the features present at the CHARA Array due to the integrated software environment. To remedy this the FLUOR software system was rewritten in the C programming language as the JouFLU server and GUI. This required an extensive ground-up conversion of the LabView code to code modeled on existing CHARA beam combiner functions. There are several major advantages to the new software system. Operations from any CHARA remote facility are now possible. JouFLU will be kept up-to-date with any system software updates to the CHARA operating environment. JouFLU data can be reduced using a modified version of the very well understood 'Classic' CHARA data reduction code. With the new software JouFLU can be integrated with other CHARA systems such as the fringe tracker, CHAMP (CHARA-Michigan Phase tracker).

\section{STATUS}

JouFLU has obtained fringes on the sky as of May 2012 and been in regular operation since 2013. Spectral dispersion mode has been tested on sky. To date, the faintest unresolved star observed with JouFLU has been FU Ori with Kmag $=5.16$ under less than optimal seeing conditions. Using the fringe contrast (visibility) SNR in these data, we estimate a point-source limiting magnitude for JouFLU of $\mathrm{K}=6$. This compares well with expectations based on the measurements of camera noise and on modeling the optical efficiency through CHARA and JouFLU. As JouFLU now moves from the engineering phase to actively collecting science data, we expect to refine this limit as data is collected on fainter targets. Work is currently underway to improve the polarization conditions within MONA which has been recently found to exhibit differential polarization rotation and phase delay. Once the difficulties of differential polarization can be resolved we expect a gain in measured visibility translating to an increase in sensitivity of approximately one magnitude.

\section{FUTURE}

There are plans to implement further upgrades for JouFLU. Plans for the near future include routine collection of spectrally dispersed fringes and to operate in FTS mode. Looking forward to the coming year, improvements will include modifications to work with a newly available feature of the CHARA Array, a fringe tracker called CHAMP. ${ }^{11}$ The addition of new optical windows will pass $\mathrm{H}$ band light to CHAMP while reflecting $\mathrm{K}$ band to JouFLU. CHAMP should enable much higher precision data to be obtained with JouFLU by reducing or removing the remaining atmospheric piston error allowing longer tracking on fringes and a higher quantity of shorter scans, increasing the data throughput, statistical precision, and overall efficiency. This should lead to higher accuracy measurements of raw visibility. Fringe tracking will also improve observation during periods of high atmospheric turbulence.

Slightly further ahead, there is a possibility of upgrading the camera to one with a PICNIC type detector within the next two years. This would operate faster and have better noise characteristics. The faster camera will enable a greater number of channels to be read for spectrally dispersed mode or faster scans for bright targets. Finally, the CHARA Array has begun work on bringing adaptive optics to the six telescopes of the interferometer. FLUOR will be among the five other beam combiners at the CHARA Array to benefit from this. The flattened wavefront provided by adaptive optics will enable light to be more efficiently injected into the fiber cores. This will allow effective use of JouFLU on nights of poor seeing that previously would have been underutilized. 


\section{CONCLUSIONS}

FLUOR was a productive and successful high precision visibility beam combiner at the CHARA Array. The upgrades of the JouFLU project have continued and enhanced that role. In addition to integrating better with the CHARA architecture, JouFLU brings higher efficiency, increased data throughput, new capabilities, greater ease of use, and more accessibility. The prospect of better alignment leading to higher throughput and faster data collection from improved stages should allow higher precision visibilities. Better methods for alignment also means that less time is lost on sky. New higher quality stepper motors increase the amount of flux incident on the fibers, increasing sensitivity. The increased speed of the raster scan decreases the amount of time spent not taking data on sky. Integration with the CHARA Array makes JouFLU accessible to a wider community of astronomers. It allows remote operation from around the world as well as rolling updates to the software control systems and data reduction software. JouFLU data compliments studies done with other beam combiners of the CHARA Array such as CLASSIC, which shares much of the same data reduction pipeline. The addition of a Fourier Transform Spectrograph mode provides a new data collection method to FLUOR and can be used to calibrate the spectral response of the NICMOS camera. The option of collecting spectrally dispersed fringes offers new possibilities for science with JouFLU. Spectrally dispersed fringes should dramatically reduce the impact of chromatic biases or bandwidth smearing. An improvement in visibility precision of a factor of 100 is expected when using spectrally dispersed fringes for cases where the science target and its calibrator are of different spectral types.

JouFLU will have a significant impact on the performance of FLUOR. While quantitative estimates of improvements at the system level are always speculative without full modeling of the instrument, our goal is to realize a threefold gain in precision and accuracy reaching $0.1 \%$ calibrated visibility amplitude measurement for a single observation bracket with multiple brackets of observation pushing this statistic lower. Once all aspects of the upgrade and the new data reduction pipeline have been optimized we expect a magnitude limit of $\mathrm{K}=6$ or fainter.

\section{ACKNOWLEDGMENTS}

The CHARA Array, operated by Georgia State University, was built with funding provided by the National Science Foundation, Georgia State University, the W. M. Keck Foundation, and the David and Lucile Packard Foundation. The CHARA Array is currently funded by the National Science Foundation under Grant AST0606958. Additional funding for the Jouvence of FLUOR upgrade project was provided by ANR EXOZODI. Research conducted with FLUOR has made use of NASA's Astrophysics Data System and of the SIMBAD database, operated at CDS (Strasbourg, France).

\section{REFERENCES}

[1] ten Brummelaar, T. A., McAlister, H. A., Ridgway, S. T., Bagnuolo, Jr., W. G., Turner, N. H., Sturmann, L., Sturmann, J., Berger, D. H., Ogden, C. E., Cadman, R., Hartkopf, W. I., Hopper, C. H., and Shure, M. A., "First Results from the CHARA Array. II. A Description of the Instrument," ApJ 628, 453-465 (July 2005).

[2] Lhomé, E., Scott, N., ten Brummelaar, T., Mollier, B., Reess, J. M., Chapron, F., Buey, T., Sevin, A., Sturmann, J., Sturmann, L., and Coudé du Foresto, V., "JouFLU: an upgraded FLUOR beam combiner at the CHARA Array," in [Society of Photo-Optical Instrumentation Engineers (SPIE) Conference Series], 8445 (July 2012).

[3] Coudé du Foresto, V., Ridgway, S., and Mariotti, J.-M., "Deriving object visibilities from interferograms obtained with a fiber stellar interferometer," A\&AS 121, 379-392 (Feb. 1997).

[4] Absil, O., di Folco, E., Mérand, A., Augereau, J.-C., Coudé du Foresto, V., Aufdenberg, J. P., Kervella, P., Ridgway, S. T., Berger, D. H., ten Brummelaar, T. A., Sturmann, J., Sturmann, L., Turner, N. H., and McAlister, H. A., "Circumstellar material in the Vega inner system revealed by CHARA/FLUOR," A $\& A$ 452, 237-244 (June 2006). 
[5] di Folco, E., Absil, O., Augereau, J.-C., Mérand, A., Coudé du Foresto, V., Thévenin, F., Defrère, D., Kervella, P., ten Brummelaar, T. A., McAlister, H. A., Ridgway, S. T., Sturmann, J., Sturmann, L., and Turner, N. H., "A near-infrared interferometric survey of debris disk stars. I. Probing the hot dust content around $\epsilon$ Eridani and $\tau$ Ceti with CHARA/FLUOR," A\&A 475, 243-250 (Nov. 2007).

[6] Akeson, R. L., Walker, C. H., Wood, K., Eisner, J. A., Scire, E., Penprase, B., Ciardi, D. R., van Belle, G. T., Whitney, B., and Bjorkman, J. E., "Observations and Modeling of the Inner Disk Region of T Tauri Stars," ApJ 622, 440-450 (Mar. 2005).

[7] Kervella, P., Coudé du Foresto, V., Traub, W. A., and Lacasse, M. G., "Interferometric Observations of the Cepheid $\zeta$ Geminorum with FLUOR / IOTA," in [Working on the Fringe: Optical and IR Interferometry from Ground and Space], Unwin, S. and Stachnik, R., eds., Astronomical Society of the Pacific Conference Series 194, 22 (1999).

[8] Bruntt, H., Kervella, P., Mérand, A., Brandão, I. M., Bedding, T. R., ten Brummelaar, T. A., Coudé du Foresto, V., Cunha, M. S., Farrington, C., Goldfinger, P. J., Kiss, L. L., McAlister, H. A., Ridgway, S. T., Sturmann, J., Sturmann, L., Turner, N., and Tuthill, P. G., "The radius and effective temperature of the binary Ap star $\beta$ CrB from CHARA/FLUOR and VLT/NACO observations," A $\& A$ 512, A55 (Mar. 2010).

[9] Mazumdar, A., Mérand, A., Demarque, P., Kervella, P., Barban, C., Baudin, F., Coudé du Foresto, V., Farrington, C., Goldfinger, P. J., Goupil, M.-J., Josselin, E., Kuschnig, R., McAlister, H. A., Matthews, J., Ridgway, S. T., Sturmann, J., Sturmann, L., ten Brummelaar, T. A., and Turner, N., "Asteroseismology and interferometry of the red giant star $\epsilon$ Ophiuchi," AEGA 503, 521-531 (Aug. 2009).

[10] Absil, O., Defrère, D., Coudé du Foresto, V., Di Folco, E., den Hartog, R., and Augereau, J.-C., "High dynamic range interferometric observations of exozodiacal discs: performance comparison between ground, space, and Antarctica," in [Society of Photo-Optical Instrumentation Engineers (SPIE) Conference Series], 7013 (July 2008).

[11] Berger, D. H., Monnier, J. D., Millan-Gabet, R., ten Brummelaar, T. A., Anderson, M., Blum, J. L., Blasius, T., Pedretti, E., and Thureau, N., "CHARA Michigan phase-tracker (CHAMP): a preliminary performance report," in [Society of Photo-Optical Instrumentation Engineers (SPIE) Conference Series], 7013 (July 2008).

[12] Fowler, A. M. and Gatley, I., "Noise reduction strategy for hybrid IR focal-plane arrays," in [Infrared Sensors: Detectors, Electronics, and Signal Processing], Jayadev, T. S. J., ed., Society of Photo-Optical Instrumentation Engineers (SPIE) Conference Series 1541, 127-133 (Nov. 1991). 